Documentation et bibliothèques

DOCUMENTATION BIBLIOTHEQUES

\title{
Technologie et millénarisme
}

Technology and Millenarianism

Tecnología y milenarismo

\section{Jacques Dufresne}

Volume 46, numéro 1, janvier-mars 2000

URI : https://id.erudit.org/iderudit/1032681ar

DOI : https://doi.org/10.7202/1032681ar

Aller au sommaire du numéro

Éditeur(s)

Association pour l'avancement des sciences et des techniques de la documentation (ASTED)

ISSN

0315-2340 (imprimé)

2291-8949 (numérique)

Découvrir la revue

Citer cet article

Dufresne, J. (2000). Technologie et millénarisme. Documentation et

bibliothèques, 46(1), 7-12. https://doi.org/10.7202/1032681ar
Résumé de l'article

L'auteur discute de la technologie et de la croyance millénariste qui s'est développée au cours de l'histoire de l'humanité, en particulier depuis les deux derniers siècles.
Tous droits réservés $@$ Association pour l'avancement des sciences et des techniques de la documentation (ASTED), 2000
Ce document est protégé par la loi sur le droit d'auteur. L'utilisation des services d'Érudit (y compris la reproduction) est assujettie à sa politique d'utilisation que vous pouvez consulter en ligne.

https://apropos.erudit.org/fr/usagers/politique-dutilisation/ 


\title{
Technologie et millénarisme
}

\author{
Jacques Dufresne \\ Philosophe
}

L'auteur discute de la technologie et de la croyance millénariste qui s'est développée au cours de l'histoire de l'humanité, en particulier depuis les deux derniers siècles.

\section{Technology and Millenarianism}

The author discusses technology and the millenarian beliefs that have developped over the course of human history, with a special focus on the last two centuries.

\section{Tecnología y milenarismo}

El autor discute la tecnología y la creencia milenarista que se ha desarrollado durante la historia de la humanidad, en particular a partir de los dos últimos siglos.
Lors du dernier Congrès ${ }^{1}$ de l'ASTED, Louis Cabral, directeur général de l'Association, a fait une déclaration digne du général de Gaulle. Durant un voyage officiel en Allemagne de l'Ouest, au début de la décennie 1960, l'éloquent général a provoqué le délire de la foule réunie à Cologne en commençant son discours dans les termes suivants: «Wir gehen nach der Zukunft ..." («Nous allons vers le futur...»). Où diable pourrions-nous aller sinon vers le futur? Et parce que nous allons vers le futur, les nouveautés finissent toujours par prendre de l'âge. D'où le lien avec la déclaration de Louis Cabral: "L'heure n'est-elle pas venue de supprimer le mot nouvelles dans l'expression nouvelles technologies?".

Pourquoi ne pas supprimer également le mot technologie? " De deux mots, il faut choisir le moindre" disait Valéry. Or, il existe un mot qui est moindre que technologie et qui signifie la même chose: technique. Pourquoi recourir au logos, qui signifie science, alors que le mot technè, qui signifie art, est une racine grecque dont notre mot technique n'a pas à rougir et qui permet, en outre, de distinguer les applications de la science elle-même.

Le mot technique a un sens clair, proche de celui du mot méthode : il y a une technique du violon, il y a une technique du béton. Comme le rappelle Jacques Ellul, on commet déjà l'erreur d'employer le mot technique à la place du mot machine ou machinisme. Pourquoi ajouter à la confusion en employant le mot technologie, encore plus général, à la place du mot technique?
Parmi les explications qu'il faut retenir, il y a l'influence de l'anglais; mais les mots anglais ayant, en l'occurrence, les mêmes racines grecques que les mots français, la même question se pose à leur sujet: pourquoi ne pas choisir le moindre?

C'est, semble-t-il, l'Américain John Bigelow, professeur à l'Université Harvard, qui a lancé le mot au milieu du XIXe siècle. Or, Bigelow était le fervent disciple de l'ingénieur John Etzler, auteur d'un best-seller paru en 1833 sous le titre de The Paradise within the reach of all men, without labor, by power of nature and machinery.

The Paradise, voici les lendemains qui chantent, voici la promesse électorale parfaite; within the reach, ce paradis sera sur terre et non dans un inaccessible audelà; all men, voici la démocratie; by power of nature and machinery, voici la technologie. Ce titre pourrait être considéré comme le thème central de la religion du progrès: le salut pour tous, mais sur terre et non dans une autre dimension, par le moyen de la technologie et non par celui de la purification personnelle. Lui-même adepte de cette religion, Bigelow était persuadé que l'homme était appelé à rétablir la domination adamique sur la nature.

Le mot technologie dans le sens qu'on lui donne depuis Bigelow désigne la technique fondée sur la science moderne par opposition aux techniques traditionnelles, celles du violoniste ou du peintre, par exemple, qui sont d'origine empirique. Mais à ce sens, qui justifie peut-être que l'on substitue le mot technologie au mot technique, s'en ajoute un second qui est proprement religieux: chaque fois qu'on utilise le mot technologie, on annonce le paradis sur terre et on adresse une prière à l'Homme, maître et souverain de la nature. II s'agit d'une parole sacramentelle.

C'est cette dimension religieuse du phénomène qui explique pourquoi en matière de technologie tant de choix ne sont pas dictés par la raison, mais par le froid calcul de ses intérêts. Quand vient le temps de s'équiper de nouvelles technologies, dans les familles comme dans les entreprises, on perd en effet la tête: rien n'est trop beau, rien n'est trop puissant. On a perdu la tête au point de commettre des erreurs aussi grossières et coûteuses que celle du bogue de l'an 2000. On estime que les quelque 500 milliards qu'aura coûté le bogue auront annulé la totalité des gains de productivité rendus possibles par les ordinateurs au cours des dernières décennies.

II n'empêche que les informaticiens continuent d'apparaître comme les sauveurs de l'humanité, alors qu'ils sont les responsables de la catastrophe. Imaginez que tous les ingénieurs du monde aient commis l'erreur de laisser subsister dans toutes les constructions en béton, y com-

1. Cette conférence a été prononcée à la fin du Congrès de l'ASTED tenu en octobre 1999. Pour bien comprendre ce texte, il faut le situer dans son contexte. Lors de la soirée d'ouverture, un monologuiste, dans le rôle d'un bricoleur en quête d'un livre sur les cabanes d'oiseaux, est venu dire comment, si l'on n'y prend pas garde, les nouvelles techniques de communication et d'information pouvaient compliquer les choses qu'elles devraient en principe simplifier. 
pris les ponts, un explosif à retardement susceptible d'exploser au cours de l'an 2000. On peut présumer que ces ingénieurs et leurs employeurs auraient été l'objet d'une poursuite judiciaire sans précédent. Au lieu d'être traités ainsi, les informaticiens reçoivent de fabuleux bonis pour réparer l'erreur qu'ils ont eux-mêmes commise. S'il en est ainsi, c'est de toute évidence parce qu'ils sont les prêtres de la nouvelle religion. On ne touche pas aux prêtres. Peut-être sont-ils considérés comme des demi-dieux ou, à tout le moins, comme les représentants de cette nouvelle espèce supérieure à l'homme animal raisonnable, le «cyborg» ou hommemachine.

À l'ouverture du Congrès, un comédien est venu dénoncer un usage des nouvelles technologies à la fois inapproprié et excessif, empreint de passion plus que de raison. Vous - membres de l'ASTED reconnaissiez par là que vous êtes vousmêmes des croisés de la religion cathodique, que vous êtes disposés à renier votre mission qui est d'être les intermédiaires entre les chercheurs et les sources d'information pour devenir les serviteurs fascinés de ce qui ne devrait être qu'un moyen comme les autres: les nouvelles technologies.

Les médias sont, le mot le dit, des intermédiaires entre l'homme et le réel. Vous êtes vous-mêmes des médiateurs et des médiatrices et vous vous avouez complices du grand mensonge du siècle agonisant: la substitution des moyens aux fins, des médias au réel dont ils sont censés nous rapprocher en s'effaçant.

Le propre des intermédiaires c'est de s'effacer. A-t-on déjà vu une entremetteuse épouser l'homme qu'elle a pour mission de jeter dans les bras de la jeune et riche héritière? Une fois qu'elle a rempli sa mission, l'entremetteuse disparaît. Les bibliothécaires ont toujours su s'effacer de la même manière. C'est ce qui faisait la grandeur discrète de votre profession. II est à craindre qu'en devenant vousmêmes «cyborgs", qu'en vous fusionnant avec les nouvelles technologies, vous ne troquiez cette grandeur contre l'avantage d'être identifiés aux sacrosaintes technologies de l'information. Déjà vous inspirez une terreur sacrée au pauvre bricoleur à la recherche d'un livre sur les cabanes d'oiseaux. Peut-être avezvous le réflexe de vous identifier à la nouvelle divinité par crainte d'être un jour rem- placés par elle. Vous auriez tort. Jamais les chercheurs, jeunes ou vieux, docteurs ou bricoleurs - la différence est-elle si grande - n'ont eu autant qu'aujourd'hui besoin d'être conduits par une main sûre vers le rayon de la bibliothèque où se trouvent les deux ou trois livres qu'ils doivent d'abord lire.

Dans le cadre du Congrès, après ma présentation de l'Encyclopédie de L'Agora (voir figure 1), j'ai eu une conversation fort éclairante à ce sujet avec une bibliothécaire, madame Bourbonnais. Elle se demandait comment elle pourrait le mieux collaborer à l'Encyclopédie. Ma réponse fut immédiate et catégorique: en exerçant votre jugement. La suite de la conversation m'a fait comprendre le dilemme qui est au cœur de votre pratique. Étant donné que vous travaillez avec des chercheurs, vous devez vous-mêmes faire preuve de la plus grande rigueur scientifique. Or, dans le contexte actuel, rigueur est synonyme de méfiance à l'égard des jugements de valeur. Votre recherche doit aussi être complète. Votre collègue chercheur ne vous pardonnerait pas d'avoir omis un ouvrage important dans votre liste. Le résultat de votre travail sera une longue liste non hiérarchisée que le chercheur auteur refilera à ses lecteurs sans la hiérarchiser davantage. Vous êtes donc partagés entre l'obligation de faire le tour d'une question et, celle plus profonde encore, mais inavouable dans le contexte actuel, d'indiquer vos préférences.

Madame Bourbonnais s'intéresse particulièrement aux personnes âgées. J'ai vite compris qu'elle pouvait dresser des listes complètes d'ouvrages sur le sujet, mais à la fin de notre conversation je connaissais aussi ses préférences. Quel est le peintre qui a le mieux présenté les personnes âgées dans son œuvre? Rembrandt. Quel est le premier livre que le non-initié devrait lire - et à plus forte raison le spécialiste, s'il ne l'a pas encore fait? Le livre de l'historien Michel Vovelle. Merci madame Bourbonnais. Rembrandt et Vovelle occuperont une place centrale dans l'Encyclopédie de L'Agora.

Et l'exercice du jugement occupera également une place centrale dans ma conférence. Kant a démontré une fois pour toutes qu'il n'y a pas de science du jugement. Trop d'imbéciles éloquents en ont conclu qu'il ne devrait pas y avoir de place pour le jugement dans la science. Tous ceux qui dans leur discipline ou leur profes- sion ont pour idéal de se conformer à ce faux principe se réduisent à la machine. Ils n'auront donc qu'à s'en prendre à euxmêmes quand on les mettra devant l'évidence qu'on peut avantageusement les remplacer par un ordinateur. La machine ne remplace que des machines. Elle ne remplit que des fonctions déjà préalablement réduites à leur aspect mécanique.

J'ai passé ma vie à faire des travaux sur des sujets divers dont je ne connaissais rien au point de départ. En 1974, nous avons à la revue Critère publié un savant numéro sur le crime. Au collège d'Ahuntsic, où j'avais mes quartiers à ce moment, on était à la fine pointe en informatique. Le directeur de la bibliothèque, de concert avec le service informatique, a produit pour nous une bibliographie d'au moins six pouces d'épaisseur. Au moment précis où j'ai pris possession de ce précieux fardeau, j'ai eu l'illusion de connaître mon sujet. Je confondais la pesanteur du paquet avec celle de mon savoir.

J'ai vite compris que dans l'ordre de la connaissance, un trésor non hiérarchisé est un paquet inutilement lourd. Et comme il nous fallait produire rapidement un numéro qui se tienne, je suis allé rencontrer la personne qui au Québec connaissait le mieux l'ensemble de la question: le professeur Henri F. Ellenberger. II m'a résumé I'histoire de la criminologie en la rattachant à d'autres disciplines, il m'a nommé ceux de ses collègues qui avaient fait les travaux les plus importants, etc.

J'ai toujours par la suite suivi la même méthode: consulter d'abord une sommité dans le domaine que je commençais à explorer. Vous me direz que tous n'ont pas cette possibilité. Je vous répondrai que documentalistes et bibliothécaires sont là pour remplacer les sommités, auxquelles la plupart n'ont pas accès directement. Quand madame Bourbonnais me recommande de lire Michel Vovelle, elle est Michel Vovelle à mes yeux.

Vous aurez compris que mon éloge du jugement est une façon de faire la critique aussi bien de la science que de la religion qui ne laissent aucune place au jugement. La religion dominante, en ce moment, c'est précisément la religion de la science, de la science et de la technologie.

Pour bien comprendre à quelles conditions l'exercice du jugement pourrait nous aider tous à échapper à l'enfermement dans la nouvelle religion, il faut évoquer l'histoire de cette dernière. Je m'inspirerai 
Figure 1. Encyclopédie de L'Agora: encyclopédie électronique créée en collaboration avec les lecteurs.

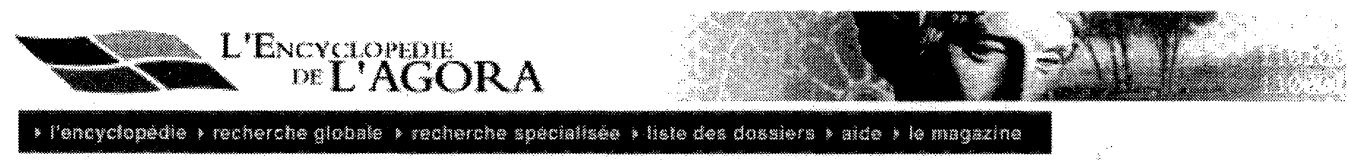

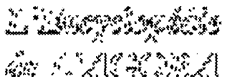

Une oeuvre évolutive et interactive. Dejà au départ, un noyau significatif de

documents, appelé à une

croissance organique, à

laquelle vous pouvez

contribuer: soit en acceptant

d'tre titulaire d'un dossier,

soit en envoyant des

documents destinés à des

dossiers déjà créés.

Plus d'informations

sur L'Encyclopédie

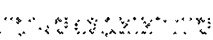

Civilisation

Culture

Médecine

Municipalité

Pour mieux comprendre le problème du regroupement

des municipalités

Sante

Liste complète 0

$\because \because \because \because \cdots$

Documents classés

par "genre de texte"

Anecdote

Anthologie

Article

Biographie

Chronologie

Citation

Commentaire

Communiqué

Conférence

Conte

Cours

Déclaration

Discours

Editorial

Entrevue

Essai

fiche de lecture

Humour

Inédit

Lettre

Liste

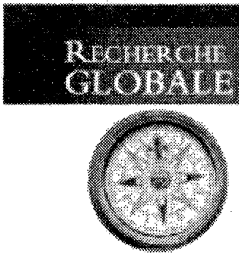

Une recherche dans le texte intégral qui donne accès à

tous les dossiers et documents contenant le mot ou la phrase

que vous inscrivez dans la

lucarne. $\mathbf{D}$

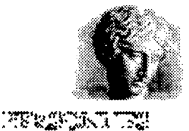

Galliée

Henri IV

Marie-Victorin

Simone Weil

Solon

Victor Hugo

Liste complète 0

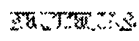

Documents classés

par "secteurs"

Administration

Affaires

Agriculture

Alimentation / Nutrition

Arimaux

Arts graphiques

Arts plastiques

Assistance sociale

Beaux arts

Cinéma

Civilisations

Commerce

Continents / Régions /

Océans

Coutumes

coutume bizarre

Croyances

Cultures / Valeurs

Eco-systèmes

Économie

Éducation

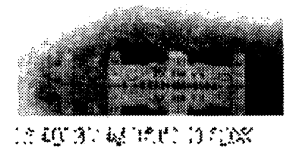

Belgique

France

Genève

Montréal

Paris

Québec - État

Liste complète $\mathbf{0}$

1.m.

Documents classés

par "disciplines"

Administration

Administration publique

Administration scolaire

Agrologie

Agronomie

Aménagement

Andragogie

Anthropologie

Archéologie

Architecture

Art

Astronomie

Astrophysique

Bibliothéconomie

Biochimie

Biologie

Biotechnologie

Botanique

Chimie

Cínématographie

Climatologie

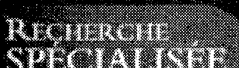

ne recherche dans les champ genre de texte, secteur, auteur, documents associés aux Animal dossiers. 0 Argent

Art

Assura

Autom

Banqu

Bibliot!

Bicycle

Bière

Biogra:

Biotecl

Bourse

Chemii

Ciném:

Comm

électro

Courrit

Crédit

Dévelo

Diction

Drogue

Eau

École

Écrivai

Eglise

Electri

Emploi

Encycl

Enfant

Enseig

Enviro

Faune

Femme

Financ

Fleur

Footba

Franco

Franco

La mode au noir améric

Réflexions hors mode sur Gastro

la mode Génét

Hélène Laberge Golf

Internet Indust

Commentaire sur un Inform

projet de Charte contre le Inforot

pillage des contenus sur Interne

le Net

Josette Lanteigne Jeunes

Internet Jeu

Une charte contre le Journ

pillage des contenus sur Livire

Voir le site <http ://agora.qc.ca> où l'on peut trouver ce texte. 
pour cela du livre de David Noble paru en 1997, The religion of technology.

Les circonstances dans lesquelles je me suis procuré ce livre ne sont pas dénuées d'intérêt. C'était à l'occasion d'un grand colloque sur le thème Education and Technology tenu à l'Université d'État de Pennsylvanie à l'automne 1997. L'organisateur de ce congrès était Carl Mitcham, un philosophe de la technique que je connaissais déjà de réputation. Parmi les participants, il y avait Ivan Illich, Neil Postman et un disciple français du regretté Jacques Ellul, Daniel Cérézuelle.

$\mathrm{Au}$ lieu de laisser aux seuls distributeurs de livres le soin d'offrir pêle-mêle des milliers de titres, les organisateurs avaient eu la bonne idée d'en mettre cinq ou six bien en évidence sur un présentoir situé juste à côté de l'auditorium. Parmi ces ouvrages élus - ne pas confondre avec bestseller - il y avait celui de David Noble. La thèse de Noble, qu'il démontre de façon assez convaincante, quoiqu'en faisant preuve d'une connaissance bien sommaire de la théologie chrétienne, c'est que l'actuelle religion de la technologie a ses racines dans le livre de l'Apocalypse et s'inscrit dans une tradition doctrinale millénaire, celle du millénarisme précisément.

David Noble rattache directement la technologie à l'histoire de la chrétienté. II ne fait pas de doute à ses yeux qu'elle est au cœur de l'une des formes de la recherche du salut qui fut adoptée par la chrétienté dès les origines, pour être rejetée dans l'ombre par la suite et reparaître au Moyen Âge: le millénarisme. Selon cette doctrine, car il s'agit d'une doctrine qui a ses racines dans la Bible, plus précisément dans l'Apocalypse de saint Jean, Dieu viendrait un jour à la tête de l'armée des bons livrer une bataille décisive aux méchants. Cette bataille, l'Armageddon, gagnée par les bons, serait suivie de mille ans de paradis sur terre.

«Puis je vis un Ange, descendre du Ciel tenant à la main la clef de l'abîme, ainsi qu'une énorme châ̂ne. Il mâ̂trisa le Dragon, l'antique serpent et l'enchaîna pour mille années. Et je vis la Cité sainte, férusalem nouvelle, qui descendait du Ciel de chez Dieu; elle s'était faite belle, comme une jeune mariée parée pour son époux. J'entendis alors une voix clamer du trône: "Voici la demeure de Dieu avec les bommes. Il aura sa demeure avec eux. Ils seront son peuple et lui, Dieu avec eux sera leur Dieu. Il essuiera toutes larmes de leurs yeux; de mort, il n'y en aura plus; de pleurs, de cris et de peine, il n'y en aura plus, car l'ancien monde s'en est allé"».

Le millénarisme, c'est l'espérance de ces mille années où le Dragon ayant été enchaîné, tous les maux, physiques et moraux, qui affligent l'humanité auront disparu, y compris la mort. Les hommes seraient alors comme des dieux dans l'état de perfection où était Adam avant le péché originel. La nature elle-même serait rétablie dans toute sa pureté et le nouvel Adam vivrait en harmonie avec elle.

La catastrophe - série de fléaux se terminant par une bataille décisive précédant immédiatement les mille années paradisiaques - a souvent été associée à la fin du monde. Chaque fois qu'on l'a crue imminente, à la fin du premier millénaire ou à l'occasion d'une grande épidémie, on a vu resurgir le millénarisme sous une forme ou une autre. La crainte d'une fin du monde prochaine n'a cependant pas toujours été liée à des catastrophes vécues ou pressenties. La condition humaine, avec son lot normal de malheurs, semble avoir suffi à maintenir en permanence un climat tel qu'il y eut toujours des gens, parmi lesquels des personnalités de premier plan, qui crurent que la fin du monde était prochaine. En conséquence, il leur fallait à la fois participer à la victoire des bons sur les méchants et la préparer par leurs travaux.

Vers l'an 1175 , le moine bénédictin, Joachim de Flore, donne ses lettres de noblesse au millénarisme. On a dit de ses prédictions qu'elles constituaient le système prophétique le plus influent que l'Occident ait connu avant Marx. Fervent lecteur de l'Apocalypse, Joachim eut une vision telle que, non seulement comprit-il mieux le passé de l'humanité, mais s'estima en mesure de prédire son avenir. Le paradis se trouvait au terme de cet avenir, et non plus hors du temps, dans une autre dimension. Dans sa marche vers ce paradis, l'humanité devait franchir trois stades correspondant aux trois personnes de la Trinité. Le premier stade, celui de Père, il l'appela ordo conjugatorium. II était caractérisé par la famille et l'état conjugal. Le second stade, celui du Fils, était appelé ordo monachorum. II avait été inauguré par saint Benoît, fondateur du premier monastère. Le troisième stade, correspondant à l'Esprit, était celui des viri spirituales, une petite élite de mâles, apparentée aux Parfaits cathares, constituant la sainte avant-garde de l'humanité rachetée. Joachim croyait que l'humanité était déjà entrée dans le troisième stade et il situait la fin du monde, c'est-à-dire l'entrée dans le millénaire bienheureux, en l'an 1260 . À ses yeux, le développement des arts mécaniques était un excellent moyen de préparer l'humanité aux lendemains qui chantent.

Ce sont les franciscains, Roger Bacon d'abord, qui prennent le relais des bénédictins millénaristes pour glorifier les arts mécaniques au XIIIe siècle.

Parmi les personnages célèbres qui par la suite seront hantés par le millénarisme, on remarque Christophe Colomb, Paracelse et surtout Tommaso Campanella. Ce dominicain né en Calabre, comme Joachim de Flore, a fomenté une révolte, vouée à l'échec, dans le but de hâter la fin de l'histoire pour créer ensuite la cité terrestre idéale. Devant les inquisiteurs qui l'interrogeaient, il reconnut qu'il estimait faire partie des viri spirituales formant ce troisième âge que Joachim avait prédit. Le paradis terrestre de Campanella s'appelait la Cité du Soleil. Le culte de la science et de la technologie était inscrit dans la charte de cette cité en tant que moyen de développement social et voie d'accès à la perfection morale.

Quant aux membres de la confrérie rosicrucienne, ils s'identifiaient aux viri spirituales; ils étaient les mâles purs à qui incombait la mission de préparer l'humanité à entrer dans le millénaire paradisiaque.

À la Renaissance, c'est l'Angleterre qui était appelée à devenir, sinon le paradis millénariste, du moins la terre où les plus grands pouvoirs seraient donnés aux adeptes de cette doctrine. On pourrait dire du millénarisme qu'il résulte d'une interprétation littérale, voire réductrice de l'Apocalypse. Libérés de l'autorité romaine et du magistère de l'Église catholique, les Anglais purent accéder directement au texte de la Bible et en faire une lecture personnelle. Le climat général favorisant la chose, plusieurs d'entre eux se passionnèrent pour l'Apocalypse et en firent une lecture semblable à celle de Joachim de Flore. Entre temps, cependant, les arts mécaniques avaient progressé au point de devenir des sciences et des techniques modernes.

L'antique croyance millénariste est toutefois restée ce qu'elle était à l'origine: 
la conviction que la fin du monde était proche et qu'il allait s'ensuivre mille années paradisiaques, auxquelles il convenait de se préparer en pratiquant le culte de la science et de la technologie. Francis Bacon, le grand « définisseur » de la modernité, John Napier, Robert Boyle, Joseph Priestley, Michael Faraday, James Clerk Maxwell, tous ces savants furent, à des degrés divers, millénaristes. Un Dieu bon, expliquait Priestley, avait créé l'univers et l'orientait dans la meilleure direction possible, celle du progrès technique. Grâce à ce progrès, l'homme allait reconquérir le pouvoir sur la nature que le péché originel lui avait fait perdre. Pour beaucoup de ces Anglais, lecteurs de l'Apocalypse, l'Amérique devait apparaître comme le lieu du paradis terrestre enfin retrouvé. Ce pays n'a d'ailleurs pas ménagé ses efforts pour éviter de les décevoir.

Nous avons déjà évoqué l'une des formes que prit le millénarisme aux ÉtatsUnis au XIXe siècle. La grande découverte que l'on fait en lisant le livre de Noble, c'est que parmi les savants américains dont on parle le plus en ce moment, ceux qui sont à la fine pointe de la recherche sur l'intelligence artificielle (IA) et sur la vie artificielle (VA), un grand nombre n'ont pas caché leur credo millénariste.

Selon Marvin Minsky, la PCO (personne en chair et en os) n'est qu'un bloody mess of organic matter. L'avenir, l'immortalité ne sont sûrement pas de ce côté. La sociologue Sherry Turkle note que les adeptes les plus enthousiastes de l'IA sont persuadés qu'une fois capables de penser par elles-mêmes et dotées d'une superintelligence, les machines vont se libérer de leurs liens avec l'organisme humain et, par là, accéder à l'immortalité.

La liste des grands prophètes de cette immortalité postbiologique et numérisée est déjà longue. J. Doyne Farmer, l'un des fondateurs du mouvement VA, intégré depuis au mouvement $I A$, ne manque pas d'audace dans ses prédictions:

"D'ici cinquante à cent ans, un nouveau type d'êtres vivants aura vraisemblablement émergé. Ces organismes seront artificiels en cè sens qu'ils auront à l'origine été conçus par des êtres bumains; ils pourront cependant se reproduire et évoluer vers des formes de vie différentes de ce $q u$ 'ils étaient à l'origine. Selon toute définition raisonnable de la vie, ils seront des êtres vivants. Ils évolueront toutefois d'une façon particulière. Le processus évo- lutif étant devenu conscient, il sera beaucoup plus rapide que par le passé.» (Cité par Turkle 1984, 353).

Earl Cox, gourou de I'IA, explique dans Beyond humanity: cyber revolution and future mind que nous vivons le déclin de la civilisation et l'aube de la supercivilisation robotique. Nous allons transférer le contenu de nos esprits dans ces vaisseaux créés par nos enfants mécaniques... Libérées de notre fragile forme humaine, ces intelligences humaines artificielles vont transcender les timides concepts de déité et de divinité tenus aujourd'hui pour vrais par les théologiens.

Daniel Crevier, autre spécialiste réputé de I'IA, soutient quant à lui, en s'appuyant sur l'Ancien et le Nouveau Testament, que l'immortalité numérique n'est pas incompatible avec le dogme chrétien de la résurrection des corps. " I/ est certain, écrit-il, que l'information et l'organisation constituant notre esprit auront besoin d'un quelconque support. Le Christ est ressuscité dans un nouveau corps; pourquoi ce nouveau corps ne serait-il pas une machine? »(Crevier 1993, 278-280)

Voilà peut-être le fin mot de la «cyberthéologie». Dans The Age of mind, transcending the human condition through robots, Hans Moravec, de l'Université Carnegie-Mellon, décrit avec précision les mécanismes de la nouvelle apothéose: «II est facile d'imaginer comment la pensée humaine pourrait se libérer de ses liens avec un corps mortel. De même, expliquet-il, que l'on peut transférer un processus de traitement de données d'un ordinateur à un autre, de même on pourrait transférer l'activité intellectuelle d'un esprit humain à un ordinateur. » (Cité par Noble 1997, 162 ). Moravec va même jusqu'à décrire l'intervention chirurgicale consistant à greffer le cerveau humain sur un ordinateur. Au fur et à mesure que le cerveau s'affaiblirait avec l'âge, l'ordinateur prendrait le relais pour remplir ses principales fonctions. Et ainsi, à condition que l'on fasse suffisamment de copies de ce logiciel personnalisé, son propriétaire d'origine serait pratiquement assuré de l'immortalité.

Michael Benedikt, président de Mental Tech inc., écrit de son côté que «Les religion sont nourries par le ressentiment que nous éprouvons à l'égard de nos corps boueux, limités, et ultime tricherie, mortels. La réalité c'est la mort. Si seulement nous le pouvions, nous pourrions al- ler de par la terre, sans jamais quitter nos maisons, vaincre sans périls, goûter aux fruits de l'Arbre sans être punis, convoler chaque jour avec des anges nouveaux, entrer au paradis, échapper à la mort. (Cité par Noble 1997, 159).

Y a-t-il beaucoup de savants contemporains plus sérieux et plus généralement respectés que John von Neuman, le père de l'ordinateur? II faut savoir qu'au moment où il s'est consacré à la cause de la guerre nucléaire préventive, il a commencé à soupeser les similitudes logiques entre la vie et la machine et à développer une théorie des automates cellulaires capables de se reproduire. Cette théorie constitue la base des recherches actuelles en VA.

Au-dessus de Von Neuman, parmi ceux que l'on considère comme les prophètes du nouveau supermonde, il y a un homme, qui fut aussi célèbre comme théologien que comme savant: Teilhard de Chardin. Au milieu du présent siècle, le théologien Teilhard de Chardin a créé le mot noosphère pour désigner l'univers d'information en train de se constituer, avec l'aide des moyens techniques, au-dessus de ce qu'on appelait déjà la biosphère. Pour beaucoup de gens, la noosphère et le virtuel constituent une même nébuleuse parée de tous les prestiges: ceux du réel aussi bien que ceux du spirituel de jadis. Le «cyborg» est la symbiose entre cette nébuleuse et le corps humain.

Teilhard compte des disciples nombreux et enthousiastes parmi les pionniers d'Internet. Le plus influent d'entre eux est le «cyber-cow-boy», John Perry Barlow. Ce que Teilhard a dit, estime Barlow, peut se résumer en une phrase simple. « Le but de toute évolution ayant eu lieu jusqu'à ce jour est la création d'un organisme collectif de l'esprit». Pour Barlow, Teilhard est le grand prophète du cyberespace. Et il commente: «'idée que le cerveau de chacun puisse s'intégrer à un réseau formé de tous les autres cerveaux, ne pouvait qu'avoir des implications théologiques pour le mystique hippie que je fus" (Cité par Freyermut 1998). « $A$ globe, clothing itself with a brain»: cette traduction anglaise d'une pensée de Teilhard est l'équivalent d'un mantra pour de nombreux internautes californiens.

L'évolution, selon Teilhard, n'est pas un phénomène purement biologique qui s'expliquerait par le hasard et la nécessité. 
Les phénomènes ont leur dehors et leur dedans. Le dedans de l'évolution c'est l'esprit, un esprit qui oriente les transformations des êtres vivants vers un degré de perfection sans cesse plus élevé. Au degré le plus élevé, Teilhard associe des mots tels que point oméga, plérôme, milieu divin. Alors que les évolutionnistes les plus audacieux avaient à peine osé imaginer un animal encore plus raisonnable, plus évolué que l'homme, Teilhard prédit un nouveau type d'évolution, une évolution de la conscience dans la noosphère, un nouveau milieu lui-même plus évolué que la biosphère dont il est issu. L'ensemble des cerveaux humains réunis par des moyens de communication assurant la simultanéité des échanges constitue la noosphère.

Le marxisme a été un millénarisme, dont les grands prêtres ont aussi pactisé allègrement avec le pouvoir, tout en obtenant du peuple qu'il renonce à tout bonheur présent, sinon à sa vie même, pour préparer les lendemains qui chantent. Et que dire des mille ans du Ille Reich! Nous découvrons grâce à Noble que le progressisme libéral et démocratique poursuit les mêmes fins.

Tel est le puissant courant par lequel nous sommes emportés vers l'immortalité terrestre et qui en attendant fait de nous des croisés de la religion cathodique. Tel est aussi la raison pour laquelle notre pauvre bricoleur ne parvient ni à obtenir un livre sur les cabanes d'oiseaux ni à établir un rapport humain avec la bibliothécaire. Cette dernière lui répond comme le ferait un robot.

II n'est pas facile de nager contre un tel courant caractérisé non seulement par le culte de la technologie, mais aussi par le prix que l'homme a dû payer pour réussir sur ce plan: la désincarnation, la montée du formalisme et par ce mal du siècle que Sifneos appelle l'alexythimie et qu'il définit comme « a condition of limited fantasy and emotional life ", un état caractérisé par l'appauvrissement de l'imaginaire et de l'affectivité.

Le remède que propose Noble me paraît être d'une portée bien limitée. Après avoir reconnu que l'utopie millénariste a été pendant longtemps un puissant facteur de réel progrès, il constate que la convergence millénaire de la technologie et de la transcendance a survécu à l'utilité historique qu'elle a pu avoir dans le passé. $\mathrm{Au}$ fur et à mesure, conclut-il, que notre entreprise technologique prend des proportions terrifiantes, il devient plus essentiel de la détacher de ses fondements religieux. À force de poursuivre l'impossible, on risque de détruire la bonne vie encore possible. C'est à cette bonne vie que Noble nous invite. Plutôt, dit-il, que de nous entêter, à coup de myriade de milliards, à créer des villes dans l'espace et à vouloir donner l'immortalité sur terre à des hommes qui n'y trouveraient qu'un incurable désespoir, pourquoi, dit-il, ne luttons-nous pas plus efficacement contre la pauvreté, pourquoi ne préférons-nous pas le petit bonheur d'occasion, le moindre moment d'un bonheur souhaité à cette vaine et froide éternité. Je cite ici un passage étonnant de Corneille, où évoquant une éternité conçue comme une durée illimitée, il écrit:

«Quand nous avons perdu le jour qui nous éclaire

Cette sorte de vie est bien imaginaire

Et moindre moment d'un bonbeur soubaité

Vaut mieux qu'une si vaine et froide éternité». (Surena, un général Parthes, tragédie)

Hélas! Noble réduit la transcendance au paradis millénariste. L'idée grecque d'une immortalité à laquelle on accède par la purification et l'idée chrétienne d'un royaume de l'amour qui n'est pas de ce monde, lui semblent totalement étrangères. L'hédonisme et l'altruisme unidimensionnels qu'il propose n'ont guère plus de sens que le paradis millénariste qu'il dénonce avec raison.

II faut répondre à la question suivante. Comment se fait-il que ce soit dans les pays dominés par la religion du Dieu incarné que les hommes se sont désincarnés au point de considérer leurs corps comme une machine et de désirer s'immortaliser sur un disque dur? On peut voir là un prétexte pour tourner le dos à cette religion. J'y vois plutôt une raison de tenter de la retrouver après sept ou huit siècles d'éloignement.

\section{Sources consultées}

Allard, Claude. 1986. L'enfant machine. Paris: Édition Balland.

Crevier, Daniel. 1993. The tumultuous history of the search for artificial intelligence. New-York:Basic Books.

Ellul, Jacques. 1982. Changer de révolution. Paris: Seuil. Hachette.

1988. Le bluff technologique. Paris:

Freyermut, Gundolf F. 1998. Die Welt, 28 mars.

Mitcham, Carl. 1994. Thinking through technology. University of Chicago Press.

Mumford, Lewis. 1950. Technique et civilisation. Paris: Seuil

1974. Le mythe de la machine. Paris: Librairie Arthème Fayard.

Noble, David. 1997. The religion of technology. New York: Knopf.

Spengler, Oswald. 1969. L'homme et la technique. Paris: Gallimard, Idées NRF.

Stefik, Mark. 1996. Internet dreams? Archetypes, myths and metaphors. London: The MIT Press.

Turkle, Sherry. 1984. The second self. New York: Simon and Shuster.

Weizenbaum, Joseph. 1981. Puissance de l'ordinateur et raison de l'homme. Paris: Éditions d'informatique.

Winner, Langdon. 1977. Autonomous Technology. London: The MIT Press. 